\title{
VARIABLES ASOCIADAS AL DETERIORO COGNITIVO EN PACIENTES DE CÁNCER DE COLON
}

\author{
VARIABLES ASSOCIATED WITH COGNITIVE IMPAIRMENT IN PATIENTS WITH \\ COLON CANCER
}

\author{
Sonia López-Santiago', Juan A. Cruzado', Ana Belén Custodio² y Jaime Feliú \\ 1 Facultad de Psicología. Universidad Complutense de Madrid \\ 2 Servicio de Oncología Médica. Hospital Universitario la Paz de Madrid
}

Resumen

Objetivo: Identificar las variables predictoras de menor rendimiento cognitivo en pacientes de cáncer de colon tratados con cirugía que esperan recibir quimioterapia.

Método: En una muestra de 89 pacientes de cáncer de colon se valoró la relación entre su rendimiento en tres dominios cognitivos (función ejecutiva, memoria verbal y habilidad psicomotora) y las siguientes variables: edad, sexo, años de escolaridad, estadio, pronóstico médico, comorbilidad, hemoglobina, ansiedad, depresión, astenia, calidad de vida, problemas físicos y psicosociales.

Resultados: En la muestra predominan los varones $(61,8 \%)$, mayores de 65 años (media 66,9), sin antecedentes neuropsicológicos $(89,9 \%)$. Los datos correlacionales ponen de manifiesto relaciones selectivas entre alguna prueba de rendimiento cognitivo y ciertas variables evaluadas. Se destaca la existencia de correlaciones negativas de la edad, los problemas respiratorios y la percepción social de la calidad de vida con el rendimiento cognitivo en pruebas de memoria verbal, función ejecutiva y habilidad psicomotora $(p<0,05)$. Así mismo, los pacientes en estadio III del cáncer de colon rinden peor que aquellos con la enfermedad en estadio II y quienes tienen menos de cinco años de estudios difieren de los de más estudios en los tres dominios cognitivos $(\mathrm{p}<0,05)$.

Conclusiones: el perfil detectado de los enfermos de cáncer de colon de alto riesgo de

\section{Abstract}

Objective: Identify predictors of lower cognitive performance in colon cancer patients treated with surgery before undergoing chemotherapy.

Methods: In a sample of 89 colon cancer patients, we evaluated the relation between their cognitive performance in three cognitive domains (executive function, verbal memory and psychomotor skill) and the following variables: Age, sex, years of study, stage, medical prognosis, comorbidity, haemoglobin, anxiety, depression, fatigue, quality of life, physical and psychosocial problems.

Results: They are a predominantly male sample $(61.8 \%)$, over 65 years (mean 66.9 ) without neuropsychological illness history $(89.9 \%)$. But, data show correlational relationships between some cognitive performance test and certain variables assessed. There are remarkable negative correlations with age, respiratory problems and social perception of the quality of life, with lower cognitive performance on tests of verbal memory, executive function and psychomotor ability ( $p$ $<0.05)$. Also, patients with stage III colon cancer performed worse than those with stage II disease and those with less than five years of study differ from most studies in the three cognitive domains $(\mathrm{p}<0.05)$.

Conclusions: The profile of patients with colon cancer at high risk of cognitive deficit is: Old age, with stage III disease, little formal education, breathing problems and a good perception of their quality of social life.

\section{Correspondencia:}

Sonia López Santiago

Alianza. Centro de Psicología. C/ Poeta Gracián n 18, $1^{\circ} \mathrm{D}$ (Granada).

E-mail: psico.lopezsantiago@gmail.com 
déficit cognitivo es: paciente mayor, con enfermedad en estadio III, de escasa formación escolar, que tiene problemas respiratorios y una buena percepción de su calidad de vida social.

Palabras clave: Cáncer de colon, rendimiento cognitivo, neuropsicología, calidad de vida, ansiedad, depresión, astenia.
Keywords: Colon cancer, cognitive performance, neuropsychology, quality of life, anxiety, depression, asthenia.

\section{INTRODUCCIÓN}

Las enfermedades oncológicas y sus tratamientos tienen un alto impacto en la vida del paciente. La ciencia avanza hacia el descubrimiento de tratamientos más selectivos, más precisos a la hora de destruir las células cancerosas, procurando a su vez reducir los efectos secundarios adversos. Para ello es imprescindible conocer la naturaleza y alcance tanto de la enfermedad como de su abordaje terapéutico y efectos secundarios asociados. La literatura científica actual señala que el Ilamado chemofog o deterioro cognitivo asociado a la quimioterapia se consolida como uno de los posibles efectos adversos de estas terapias sistémicas.

La necesidad de su estudio se basa en la relevancia del funcionamiento de las capacidades cognitivas para el desenvolvimiento óptimo de la persona en su vida diaria. Sobre el análisis de esta cuestión hay que señalar que algunos trabajos científicos, de diseño transversal, corren el riesgo de sobreestimar el daño cognitivo quimio- inducido, pues no tienen en cuenta el deterioro previo achacable a otras variables existentes antes de la administración de dicha terapia sistémica. Hasta tal punto es importante realizar una evaluación previa al tratamiento, que algunos autores destacan la necesidad de contar con métodos de screening, que detecten los posibles casos de una forma breve y eficaz (Vardy et al. ${ }^{(1)}$ con una muestra de 31 pacientes, compuesta predominantemente por mujeres con cáncer de mama, detectaron entre un 26 y $55 \%$ de deterioro cognitivo de moderado a severo con diferentes pruebas de screening).

Algunos estudios confirman la existencia de daños cognitivos previos a la administración de quimioterapia, este es el caso de los siguientes trabajos: Rodin et al. ${ }^{(2)}$, en un estudio realizado en una muestra de 19 mujeres mayores de 50 años con cáncer de mama, detectan un $37 \%$ de deterioro cognitivo significativo; Wefel, Saleeba, Buzdar y Meyers ${ }^{(3)}$ encontraron un $21 \%$ de deterioro previo al tratamiento (9 de 42 mujeres con cáncer de mama) sobre todo en aprendizaje y memoria, función ejecutiva y velocidad de procesamiento. Meyers, Byrne y Komaki(4), en cáncer de pulmón también hallaron déficits en memoria verbal, función ejecutiva y coordinación motora antes de recibir el tratamiento de quimioterapia y radioterapia.

Diversos factores personales o ambientales podrían estar influyendo en estos resultados. Por ello, este trabajo tiene el objetivo de valorar las variables predictoras del funcionamiento cognitivo en pacientes de cáncer de colon tras la cirugía y previa administración de quimioterapia.

\section{MÉTODO}

\section{Muestra}

Los participantes forman parte de un estudio longitudinal en curso. El equipo médico del Servicio de Oncología Médica del Hospital Universitario La Paz de Madrid reclutó a los pacientes que cumplían 
un conjunto de requisitos de inclusión y no cumplían ninguno de los criterios de exclusión (ver tabla 1). Las características de la muestra configuran el siguiente perfil: - Datos sociodemográficos: varón $(61,8 \%)$ de 67 años (media $=66,9 ;$ Dt = 9,19; rango 35-85; el 52,2\% tienen entre 50 y 69 años y el 43,3\% más de 70 años), casado (80,9\%), con hijos $(85,5 \%)$; vive en pareja $(75 \%)$; dominancia manual diestra (93,2\%).

- Escolaridad y trabajo: el 34,4\% de los participantes tiene menos de cinco años de estudios, y el $65,6 \%$ restante tiene entre cinco y doce años de estudios o más, aunque el 5452,3\% no llegó a alcanzar la titulación de EGB; trabajó por cuenta ajena $(40 \%)$ y actualmente está jubilado (53,9\%).

- Antecedentes clínicos: no presenta antecedentes de enfermedad neuropsicológica (89,9\%), el nivel de hemoglobina basal es de 12,4 g/dl. (media en hombres de 12,65 y en mujeres de 11,96 g/ dl.) y no presenta historial oncológico previo $(85,2 \%)$ ni otras enfermedades crónicas $(57,3 \%)$.

- Trastornos actuales: no refiere trastornos de salud mental $(87,6 \%)$ y comunica no tener problemas físicos relacionados con la enfermedad o con los tratamientos ni problemas psicosociales de gravedad. A nivel de afectados, destaca una astenia moderada, que padece el $21 \%$ de la muestra, seguida de problemas digestivos, característicos de la enfermedad oncológica padecida. De igual modo, los pacientes refieren sentimientos de tristeza (el 22,5\%), seguidos de problemas de sueño y ansiedad, con intensidades moderadas, que alcanzan una puntuación de 9 sobre 10 en algún caso. No necesita cuidador (93,3\%).

- Situación clínica actual: diagnosticado de cáncer de colon, estadio III (61\%), siendo el la masa afecta resecada mediante sigmoidectomía o hemicolectomía derecha tiempo desde la cirugía hasta que recibe quimioterapia oscila entre 2 y 8 semanas. Recibirá FOLFOX-4 o XELOX $(95,1 \%)$. La terapia tiene una duración promedio de seis meses, independientemente del régimen administrado. Su pronóstico es favorable $(62 \%)$.

\section{Variables e instrumentos de medida}

La tabla 2 muestra los datos referentes a los dominios cognitivos y los instrumentos de evaluación aplicados para su evaluación. La tabla 3 recoge las variables moduladoras de interés y las pruebas de evaluación correspondientes.

\section{Tabla 1. Criterios de inclusión y exclusión}

\begin{tabular}{|c|c|}
\hline CRITERIOS DE INCLUSIÓN & CRITERIOS DE EXCLUSIÓN \\
\hline Mayor de 18 años & Menor de 18 años y mayor de 85 años \\
\hline Cáncer de colon estadios II y III & $\begin{array}{c}\text { Estadio de enfermedad: } \\
\text { I: no requiere quimioterapia } \\
\text { IV: metástasis actuales }\end{array}$ \\
\hline $\begin{array}{c}\text { Prescripción de quimioterapia } \\
\text { (combinado con oxaliplatino) }\end{array}$ & $\begin{array}{c}\text { Tratamiento sistémico anticáncer conco- } \\
\text { mitante }\end{array}$ \\
\hline Consentimiento informado & Adicción a sustancias \\
\hline $\begin{array}{c}\text { Comprensión y expresión adecuada del } \\
\text { castellano }\end{array}$ & $\begin{array}{c}\text { Enfermedad neurológica o trastorno psi- } \\
\text { quiátrico grave }\end{array}$ \\
\hline
\end{tabular}




\section{Tabla 2. Variables dependientes}

\begin{tabular}{|c|c|c|}
\hline $\begin{array}{l}\text { Variable depen- } \\
\text { diente: }\end{array}$ & Operativamente & Pruebas de Evaluación. \\
\hline $\begin{array}{c}\text { Memoria } \\
\text { verbal }\end{array}$ & $\begin{array}{l}\text { - Número de ítems verbales en } \\
\text { recuerdo inmediato, con y sin } \\
\text { indicios. } \\
\text { - Número de ítems verbales en } \\
\text { recuerdo diferido, con y sin } \\
\text { indicios. } \\
\text { - Máximo número de palabras } \\
\text { aprendidas de un listado. }\end{array}$ & $\begin{array}{l}\text { Memoria de textos del Test Barce- } \\
\text { lona }{ }^{(5-8)} \text {. (Test Barcelona. } \\
\text { Peña-Casanova, 1990). } \\
\text { Memoria de textos del Test Bar- } \text { celona }^{(5-8)} \text { (Test Barcelona. Peña- } \\
\text { Casanova, 1990). } \\
\text { Aprendizaje seriado de palabras } \\
(\text { Test Barcelona. Peña-Casanova, } \\
\text { 1990) })^{(5-8)} \text {. }\end{array}$ \\
\hline $\begin{array}{l}\text { Función } \\
\text { ejecutiva }\end{array}$ & $\begin{array}{l}\text { - Proceso de interferencia cogni- } \\
\text { tiva. } \\
\text { - Coordinación de categorías para } \\
\text { realizar una tarea. }\end{array}$ & $\begin{array}{l}\text { Stroop, test de colores y palabras } \\
{\text { (Golden, } 1975)^{(9)} \text {. }} \\
\text { Trail Making Test B (Partington, } \\
\text { 1949) })^{(10-12)} \text {. }\end{array}$ \\
\hline $\begin{array}{c}\text { Función psi- } \\
\text { comotora }\end{array}$ & $\begin{array}{l}\text { - Copiar símbolos rápidamente. } \\
\text { - Velocidad y adecuación de } \\
\text { trazado. }\end{array}$ & $\begin{array}{l}\text { Clave de números (Test Barcelo- } \\
\left.\text { na. Peña-Casanova, } 1990^{(5-8)}\right) \text {. } \\
\text { Trail Making Test A (Partington, } \\
\text { 1949)(10-12). }\end{array}$ \\
\hline
\end{tabular}

\section{Análisis de datos}

Se llevó a cabo mediante el programa estadístico SPSS versión 19.0. Se obtuvieron estadísticos descriptivos tales como la media, la mediana, máximos y mínimos, porcentajes y frecuencias.

Se utilizó la correlación de Pearson para variables que cumplían propiedades de aplicación de pruebas paramétricas y correlación de Spearman para las que no.

Para las diferencias intergrupales se utilizaron las siguientes pruebas:

- Para dos muestras independientes (diferencias según género y estadio): la prueba $t$ de Student cuando las variables cumplían las propiedades de aplicación de pruebas paramétricas y Mann-Whitney para las que no.
- Para más de dos muestras independientes (diferencias según años de estudios, pronóstico médico y comorbilidad): la prueba de ANOVA para las diferencias de medias junto con HSD de Tukey para las comparaciones múltiples en las variables que cumplían los requisitos para aplicación de pruebas paramétricas y Kruskall-Wallis para las que no.

\section{Procedimiento}

Este trabajo forma parte de un estudio prospectivo en curso. Los investigadores elaboraron un proyecto de investigación que fue evaluado y aceptado por el comité ético del Hospital Universitario La Paz. Posteriormente, el equipo médico del 
Tabla 3. Variables moduladoras

\begin{tabular}{|c|c|c|}
\hline Variables moduladoras. & Operativamente & Instrumento de evaluación \\
\hline Sociodemográficas & $\begin{array}{l}\text { Edad, género, años de } \\
\text { educación. }\end{array}$ & $\begin{array}{l}\text { Cuestionario sociodemo- } \\
\text { gráfico }\end{array}$ \\
\hline Clínicas & $\begin{array}{l}\text { Estadio (II o III) } \\
\text { Pronóstico (muy favorable, } \\
\text { favorable, dudoso, des- } \\
\text { favorable) Comorbilidad } \\
\text { (sólo cáncer, una adicio- } \\
\text { nal, más de una enferme- } \\
\text { dad adicional) } \\
\text { Problemas físicos y psico- } \\
\text { sociales asociados (intensi- } \\
\text { dad 1-10) } \\
\text { Antecedentes de enferme- } \\
\text { dad (oncológica, neuropsi- } \\
\text { cológica) }\end{array}$ & Entrevista estructurada \\
\hline $\begin{array}{l}\text { Calidad de vida: } \\
\text { - Status global } \\
\text { - Áreas funcionales: } \\
\text { Física. Social. Rol } \\
\text { Emocional. Cognitiva } \\
\text { - Escalas sintomáticas: } \\
\text { Astenia. Náuseas. Dolor } \\
\text { - Síntomas específicos: } \\
\text { Disnea. Insomnio. Apetito } \\
\text { Estreñimiento. Diarrea } \\
\text { Economía }\end{array}$ & $\begin{array}{l}\text { Puntuación dada a dife- } \\
\text { rentes ítems que evalúan } \\
\text { síntomas, funciones de } \\
\text { calidad de vida y puntua- } \\
\text { ción global }\end{array}$ & $\begin{array}{l}\text { Escala QLQ-C30 de } \\
\text { EORTC (1993). } \\
\text { Versión española, Arra- } \\
\text { rás, Illarramendi y Valerdi } \\
(1996)^{(13-16)}\end{array}$ \\
\hline $\begin{array}{l}\text { Ansiedad } \\
\text { Depresión }\end{array}$ & $\begin{array}{l}\text { Puntuación dada a ítems } \\
\text { de ansiedad y depresión }\end{array}$ & $\begin{array}{l}\text { HAD (Zigmond y Snaith, } \\
\text { 1983). Versión española, } \\
\text { adaptación de Caro e lbá- } \\
\text { ñez }(1992)^{(17-18)}\end{array}$ \\
\hline Astenia & $\begin{array}{l}\text { Puntuación en ítems de } \\
\text { astenia general e interfe- } \\
\text { rencia }\end{array}$ & $\begin{array}{l}\text { Inventario de Fatiga Breve } \\
\text { Universidad de Texas MD. } \\
\text { Anderson Cancer Center. } \\
\text { (Cleeland, 1998) }{ }^{(19-20)}\end{array}$ \\
\hline Hemoglobina & $\begin{array}{l}\text { Anemia: } \\
<12 \mathrm{~g} / \text { dl. en mujeres } \\
<13 \mathrm{~g} / \mathrm{dl} \text {. en hombres }\end{array}$ & Hemograma \\
\hline
\end{tabular}


Servicio de Oncología Médica procedía al reclutamiento de los pacientes que cumplían los criterios de inclusión y ninguno de los de exclusión. A quienes aceptaron colaborar, se les daba una cita, en la cual se extraía una muestra de sangre, se aportaba información médica pertinente al tratamiento quimioterapéutico y se procedía a la aplicación de un protocolo de evaluación que incluía tanto pruebas neuropsicológicas como de autoinforme.

\section{RESULTADOS}

Los resultados correlacionales y comparativos de variables moduladoras de la función cognitiva se exponen a continuación:

\section{Edad}

Existen correlaciones significativas en rendimiento cognitivo según la edad del paciente oncológico. Concretamente, hay una correlación negativa estadísticamente significativa entre la variable edad y las puntuaciones en las pruebas Clave de números $(r=-0,65 ; p=0,000)$, Aprendizaje seriado de palabras $(r=-0,48 ; p=$ $0,000)$, subpruebas del test Stroop (Palabra: $r=-0,33 ; p=0,002$; Color: $r=-0,31$; $p=0,004)$, y las subpruebas de Recuerdo de textos (Inmediato libre: $r=-0,37, p=$ 0,000; Inmediato con indicios: $r=-0,24$, $p=0,032$; Diferido libre: $r=-0,28, p=$ 0,011 y Diferido con pistas: $\rho=-0,30, p$ $=0,003)$. Existe una correlación positiva significativa entre la edad y las puntuaciones en el TMT-A $(\rho=0,59 ; p=0,000)$ $y$ en el TMT-B $(r=0,26 ; p=0,014)$, esto revela que los pacientes mayores invierten más segundos en resolver estas dos tareas, obteniendo peores resultados. No hubo correlación significativa $(p>0,05)$ entre la edad y las puntuaciones en ColorPalabra ni con el factor Interferencia del test Stroop. La mayor parte de los datos respalda la hipótesis de que el rendimiento cognitivo empeora con la edad.

\section{Años de escolaridad}

Para valorar las diferencias en funcionamiento cognitivo según el grado de escolaridad, se realizó un ANOVA tomando como variable independiente los años de estudios realizados con tres niveles: alto (participantes con mas de doce años de escolaridad), medio (entre cinco y doce años de estudios) y bajo (menos de cinco años de estudios). El recuerdo diferido con indicios y el TMT-A y B incumplieron los supuestos para aplicar pruebas paramétricas.

El ANOVA reveló diferencias en las pruebas en las que fue aplicado salvo en el Factor de Interferencia. A continuación se utilizó la prueba de Tukey para discernir entre qué subgrupos había diferencias significativas (bajo, medio y alto). Se apreciaron diferencias significativas entre los pacientes de status académico medio y alto $(p<0,05)$, excepto en Aprendizaje seriado de palabras y Recuerdo Diferido libre. Existen diferencias significativas $(p<$ $0,01)$ entre el nivel de instrucción bajo y el alto, quienes tienen menos de cinco años de estudios rinden peor. Además, se detectó un menor rendimiento en los pacientes de cáncer de colon cuya escolaridad no supera los cinco años de enseñanza académica frente a los que tienen entre cinco y doce años de estudios salvo en Color, Color-Palabra ( $p>0,05)$.

En los casos que no cumplían los criterios para aplicación del ANOVA se realizó la prueba no paramétrica de Kruskal-Wallis, que confirmó la existencia de diferencias significativas por años de educación en el TMT-A $(\chi 2=22,35 ; p=0,000)$, en el TMT$B(\chi 2=28,26 ; p=0,000)$, $y$ también en la subprueba Recuerdo Diferido de historias con indicios $\left(\chi^{2}=25,01 ; p=0,000\right)$. Los datos muestran una tendencia en la que 
menos años de instrucción académica se corresponden invariablemente con peor rendimiento neuropsicológico del paciente.

\section{Sexo}

Se valora la existencia de un funcionamiento cognitivo diferencial por sexo entre los 54 varones y las 32 mujeres del estudio que cumplimentaron todas las pruebas. Las comparaciones estadísticas se realizaron mediante pruebas paramétricas (prueba t) y no paramétricas (U de Mann-Whitney); los test neuropsicológicos que no cumplen criterios para emplear pruebas paramétricas son el TMT-A y el Recuerdo Diferido con indicios. Los resultados obtenidos con ambas pruebas, paramétricas y no paramétricas, reflejan ausencia de diferencias estadísticamente significativas según sexo en función ejecutiva, memoria verbal y habilidad psicomotora. Se acepta la hipótesis nula dado que $\mathrm{p}>0,05$.

\section{Ansiedad}

Según las puntuaciones en la Escala de Ansiedad y Depresión Hospitalaria (HAD), los pacientes de cáncer de colon muestran un promedio de 4,6 sobre 21 puntos en la escala ansiedad (Dt $=3,44$ y rango $0-17$ ). El $82 \%$ de los pacientes (73 participantes) obtuvieron puntuaciones dentro del rango de la normalidad, el 11,2\% se calificó como caso dudoso (10 personas) y el $6,6 \%$ (6 personas) alcanzó un resultado clínico en ansiedad. Los análisis correlaciónales indican ausencia de relación significativa entre la ansiedad medida con el HAD y el rendimiento neuropsicológico $(p>0,05)$.

\section{Depresión}

Según las puntuaciones en la Escala HAD, los pacientes de cáncer de colon muestran un promedio de depresión de 3,8 sobre 21 puntos (Dt $=3,57$ y rango $0-18$ ) Con anterioridad a la administración de quimioterapia, el 80,9\% (72 participantes) de los pacientes presentó puntuaciones en la escala depresión dentro del rango considerado normal (0-7), el 12,4\% (11 personas) fueron calificados como caso dudoso, y el $6,6 \%$ de la muestra (6 personas) obtuvo una puntuación clínica en dicha escala. Los datos indican ausencia de relaciones estadísticamente significativas entre el grado de depresión medido con el HAD y el rendimiento en las diferentes pruebas administradas $(p>0,05)$.

\section{Astenia}

Mediante el Inventario Breve de Fatiga (BIF) se detecta que la muestra obtiene una astenia media general de 2,58 sobre 10 (Dt $=2,63$ y rango $0-8$ ), mientras que la media de la interferencia de ésta en diferentes aspectos de la vida diaria es de 0,73 (Dt $=1,46$ y rango 0-8). Ambas puntuaciones se consideran bajas. Además, no existe correlación estadística significativa $(p>$ $0,05)$ entre el rendimiento cognitivo y las puntuaciones en astenia (nivel de astenia general e interferencia con áreas de la vida del paciente).

\section{Nivel basal de hemoglobina en sangre}

La media grupal es de 12,41 g/dl. (Dt $=1,48$ y rango $9,1-15,6)$. La media en los hombres $(n=45)$ es de $12,65 \mathrm{~g} / \mathrm{dl}$. y de $11,96$ en las mujeres ( $n=24)$, ambos resultados reflejan anemia. Un 53\% de los hombres y un $46 \%$ de las mujeres presentan anemia. Únicamente la prueba TMT-A correlaciona negativamente con la hemoglobina de la muestra de forma significativa ( $\rho=-0,30 ; p=0,01)$. Cuanto menor es la concentración de hemoglobina en sangre más segundos empleados en terminar la prueba, afectando a la velocidad psicomotora. 


\section{Pronóstico médico}

Se procedió a hallar las diferencias grupales mediante el ANOVA para variables paramétricas, y la prueba Kruskal-Wallis para variables no paramétricas. Se acepta la hipótesis nula, pues no hubo diferencias significativas intergrupos $(p>0,05)$.

\section{Comorbilidad}

Se emplean pruebas paramétricas (ANOVA) y no paramétricas (KruskallWallis). Puntualmente, la prueba Aprendizaje seriado de palabras presenta diferencias estadísticamente significativas según la comorbilidad del paciente $(p=0,02)$. La prueba HSD de Tukey pone de manifiesto diferencias estadísticamente significativas entre el grupo con cáncer y los que tienen una enfermedad crónica adicional, con una diferencia de medias de 8,09 y sig. $=0,041$.

\section{Estadio del cáncer de colon}

A continuación se muestran los datos paramétricos (ofrecidos por la prueba t) y no paramétricos ( $U$ de Mann-Whitney) sobre las diferencias en funcionamiento cognitivo según el estadio de la enfermedad oncológica en la muestra, II ó III:

A excepción de la interferencia $(t=$ $1,36 ; p=0,17)$, aparecen diferencias estadísticamente significativas en rendimiento cognitivo a favor de la hipótesis alternativa y siempre en la misma dirección. Los participantes en estadio III de la enfermedad tienen una peor puntuación que los participantes en estadio II en: Clave de números $(\mathrm{t}=2,42 ; \mathrm{p}=0,01)$, TMT-A ( $\mathrm{Z}=$ $-2,83 ; \mathrm{p}=0,005)$, TMT-B $(\mathrm{t}=-2,17 ; \mathrm{p}=$ $0,03)$, subpruebas del test Stroop: Palabra $(\mathrm{t}=2,81 ; \mathrm{p}=0,006)$, Color $(\mathrm{t}=2,35 ; \mathrm{p}=$ $0,002)$, Color-Palabra ( $t=2,95 ; p=0,004)$, Aprendizaje seriado de palabras $(t=3,07$; $p=0,003)$, Recuerdo de Historias Inme- diato Libre $(t=2,44 ; p=0,01)$, Inmediato con indicios $(t=3,08 ; p=0,003)$, Diferido Libre $(t=3,42 ; 0,001)$ y Diferido con indi$\operatorname{cios}(Z=-3,09 ; p=0,002)$.

El análisis de casos de deterioro clínico según estadio, II o III, muestra que en función ejecutiva el porcentaje promedio de pacientes en estadio II con deterioro es de $23 \%$ (borderline $15,2 \%$ y grave $7,78 \%$ ), mientras, en los pacientes con cáncer en estadio III, el porcentaje de casos deteriorados es de $41 \%$ (deterioro sutil $23,84 \%$ y grave $17,28 \%$ ). En el dominio de memoria verbal, un $3,12 \%$ de los pacientes con enfermedad en estadio II ostenta un deterioro calificable como borderline; frente al 13\% de deterioro de aquellos que alcanzan el estadio III (borderline 10,46\% y grave $2,6 \%$ ). En cuanto a los casos clínicos encontrados en el dominio cognitivo habilidad psicomotora, aquellos con cáncer en estadio II presentan un $4,65 \%$ de deterioro sutil, por su parte, en estadio III se observa deterioro en el $29 \%$ de los participantes (sutil $27,1 \%$ y grave en la prueba TMT-A un $2,1 \%)$.

Teniendo en cuenta el rango de deterioro, siendo el valor mínimo el correspondiente al dominio con más casos de deterioro (función ejecutiva), en el supuesto de que estos mismos pacientes también muestran algún daño en los otros dos dominios, y el valor máximo correspondiente al sumatorio del deterioro promedio en los tres dominios, en el supuesto de que diferentes pacientes pueden tener daño en diferentes dominios. El rango de deterioro en estadio II oscilaría entre el $23 \%$ y $31 \%$; mientras que en estadio III puede oscilar entre 41 y $83 \%$. Se observa más del doble de deterioro en pacientes con enfermedad en estadio III. En aquellos con estadio III se aprecia que el daño cognitivo predomina sobre todo en función ejecutiva, seguido de habilidad psicomotora y de memoria verbal en menor medida. 


\section{Calidad de vida}

En cuanto a la calidad de vida global, mantiene una correlación positiva significativa con Aprendizaje seriado de palabras $(r=0,25, p=0,017)$. En el resto de pruebas se acepta la hipótesis nula por ausencia de correlaciones estadísticamente significativas

En relación a las cinco áreas funcionales de la calidad de vida, se realizaron correlaciones no paramétricas mediante la prueba de Spearman. Las áreas física, rol, emocional y cognitiva no correlacionan significativamente con las pruebas cognitivas.

Únicamente se aprecia que la función social se relaciona con algunas pruebas y subpruebas de algunos test (Clave de números: $\rho=-0,38 ; p=0,000$; TMT-A: $\rho$ $=0,32 ;$ y $p=0,002$; TMT-B: $\rho=0,25$; $\mathrm{p}=0,02$; subpruebas $\mathrm{P}$ del test Stroop: $\rho$ $=-0,29 ; p=0,006$ y $C: \rho=-0,24 ; p=$ 0,02; Aprendizaje seriado de palabras: $\rho=$ $-0,21 ; p=0,04$; recuerdo inmediato libre: $\rho=-0,31 ; p=0,003$; inmediato con indicios $\rho=-0,27 ; p=0,009$; diferido libre $\rho=$ $-0,23 ; p=0,02$ y diferido con indicios $\rho=$ $-0,25 ; p=0,01)$. Esta relación se establece en una dirección contraria a lo esperado, a un mayor apoyo social percibido le corresponde un menor rendimiento en las pruebas.

Se acepta la hipótesis nula planteada entre el rendimiento en las pruebas y las quejas del paciente respecto a problemas de concentración y memoria $(p>0,05$ en las correlaciones entre funcionamiento cognitivo y área funcional cognitiva de la calidad de vida).

Existe una correlación significativa entre la percepción subjetiva cognitiva y el estado de ánimo (Depresión: $\rho=-0,44 ; p$ $=0,000$; Ansiedad $\rho=-0,42 ; p=0,001)$. Una mayor puntuación en sintomatología ansiosa y depresiva se relaciona con más quejas en memoria y concentración.

\section{Screening sobre problemas físicos percibidos por el paciente}

Los problemas relacionados con la respiración constituyen una variable física que mantiene relaciones estadísticamente significativas con la mayor parte de las pruebas neuropsicológicas empleadas (excepto: Interferencia $p>0,5)$. Existe una correlación positiva con TMT-A $(\rho=0,34 ; p=$ $0,001)$ y TMT-B $(\rho=0,22 ; p=0,03)$ y correlaciones negativas significativas con: Clave de Números $(\rho=-0,35 ; p=0,001)$, test Stroop: subpruebas Palabra $(\rho=-0,32 ; p=$ $0,002)$, Color $(\rho=-0,24 ; p=0,02)$, ColorPalabra $(\rho=-0,32 ; p=0,002)$, Aprendizaje seriado de palabras $(\rho=-0,34 ; p=0,001)$; Recuerdo de Historias: Recuerdo Inmediato Libre $(\rho=-0,29 ; p=0,004)$, Inmediato con indicios $(\rho=-0,27 ; p=0,009)$, Diferido Libre $(\rho=-0,29 ; p=0,006)$ y Diferido con indicios $(\rho=-0,28 ; p=0,006)$.

Puntuaciones mayores en dolor se relacionan significativamente con peor Recuerdo Diferido de textos con o sin indicios $(\rho=-0,24$ y $p=0,02$ en ambos). La fatiga referida por el paciente se asocia a peor rendimiento en el TMT-A ( $\rho=22$; $p$ $=0,03)$ y en Recuerdo Inmediato libre y Diferido con indicios ( $\rho=-24$ y $\rho=-0,26$; $p<0,05$ en ambos casos).

\section{Screening sobre problemas psicosociales actuales percibidos por el paciente}

El test Dígito-símbolo presenta una correlación positiva con problemas de administración del tiempo y solución de problemas $(\rho=0,23 ; p=0,02)$.

El TMT-A no correlaciona con este tipo de problemas.

Una mejor puntuación en el TMT-B se relaciona significativamente con duelo en el paciente $(\rho=-0,22 ; p=0,03)$.

Un menor Aprendizaje seriado de palabras se asocia con mayores problemas de 
sueño $(\rho=-0,25 ; p=0,02)$ y con la dependencia en el paciente $(\rho=-0,22 ; p=0,03)$.

En el caso del test Stroop, ninguno de los aspectos que evalúa mantiene relación con los problemas psicosociales.

En Recuerdo de Historias se aprecian diversas correlaciones significativas:

- El recuerdo Inmediato con indicios COrrelaciona positivamente con otros problemas $(\rho=0,21 ; p=0,04)$.

- El Recuerdo Diferido de textos sin pistas mantiene una correlación negativa significativa con la ansiedad referida por el paciente $(\rho=-0,23 ; p=0,02)$ y con déficit de actividades de ocio $(\rho$ $=-0,27 ; p=0,01)$. En contra de lo esperado, mantiene una relación positiva significativa con otros problemas psicosociales $(\rho=0,21 ; p=0,04)$.

- El recuerdo Diferido con indicios correlaciona de forma estadísticamente negativa con sentimientos de ansiedad $(\rho=-0,23 ; p=0,03)$.

Existen datos que indican que a mayor problemática psicosocial menor será el rendimiento del paciente en algunas pruebas cognitivas. Pero hay correlaciones en sentido opuesto. Ciertos problemas psicosociales se relacionan con un peor rendimiento neuropsicológico en las pruebas mientras que otros se relacionan con mejor rendimiento.

\section{DISCUSIÓN}

El estudio cuenta con una muestra de 89 participantes compuesta principalmente por varones mayores de 50 años, que a sus más de 65 años están jubiladas, en su mayoría no tienen antecedentes de enfermedad neurológica. Un 34,4\% de los participantes tiene menos de cinco años de estudios, no cuentan con el factor de protección de deterioro neuropsicológico que confiere una escolaridad elevada. Predominan los pacientes en estadio III del cáncer, operados mediante sigmoidectomía o hemicolectomía derecha, con pronóstico favorable, y con prescripción médica de FOLFOX-4 durante un periodo de tiempo de 6 meses. Un 35\% de los hombres y un $46 \%$ de las mujeres tienen anemia. Comunica no tener problemas físicos ni psicosociales de elevada gravedad. No obstante existen algunos casos con problemas de elevada intensidad (sobre todo astenia y sentimientos de tristeza) que haría recomendable su evaluación pormenorizada y tratamiento.

Este trabajo se centra en valorar las variables que predicen el rendimiento cognitivo en función ejecutiva, memoria verbal y habilidad psicomotora en una muestra de pacientes de cáncer de colon tratados con cirugía antes de recibir quimioterapia.

Los datos confirman que los pacientes de mayor edad son los que peor ejecutan las pruebas neuropsicológicas propuestas. Sin embargo, el rendimiento en la liberación de la interferencia cognitiva no mantiene correlaciones significativas. Las puntuaciones del test Stroop fueron corregidas por la edad, por lo que la independencia entre ambas variables es lógica.

Queda contrastado, que los años de escolaridad influyen positivamente en las pruebas neuropsicológicas; los pacientes que tienen menos de cinco años de estudios tienen un rendimiento inferior al en los tres dominios cognitivos.

La influencia de la edad y los años de escolaridad pone de relieve que las personas mayores y quienes tienen una escasa experiencia académica son más vulnerables a déficit cognitivos. La conjunción de ambos factores haría que esos pacientes oncológicos pudieran ser más sensibles a agentes perjudiciales para su capacidad mental en algunas facetas cognitivas.

Un estudio precedente constató un peor rendimiento en hombres en los test clásicos $^{(21)}$. Este estudio no confirma tales conclusiones porque los datos respaldan una ausencia de diferencias significati- 
vas en el funcionamiento cognitivo según sexo. Resulta recomendable que próximos estudios contemplen esta variable para contrastar estos datos contradictorios.

Pese a que era de esperar que la ansiedad y la depresión (evaluadas mediante el HAD) perjudicasen el funcionamiento neuropsicológico, esta investigación no lo confirma. Un estudio señaló que, la conocida relación de estas variables con un peor rendimiento cognitivo, no puede ser ratificada cuando un elevado porcentaje de la muestra presenta valores normales de ansiedad y depresión ${ }^{(22)}$.

Los pacientes se sometieron a tratamiento quirúrgico entre uno y dos meses antes de realizar la evaluación neuropsicológica, lo cual hace probable una atenuación progresiva de la astenia (media inferior a tres puntos sobre 10). El escaso padecimiento de astenia podría no ser suficiente para perjudicar el rendimiento cognitivo.

De igual modo, la hemoglobina sólo correlaciona negativamente de forma significativa con el TMT-A, los participantes que han invertido más segundos para completar esta prueba presentan un valor de hemoglobina más bajo. La presencia de anemia en el organismo podría ralentizar la velocidad en la realización de la tarea.

Resaltar que el rendimiento en los test neuropsicológicos no se asocia al pronóstico médico del paciente, ni la mayoría de las pruebas se relacionan con la comorbilidad (la presencia de varias enfermedades crónicas adicionales al cáncer sólo repercute negativamente en su capacidad para el aprendizaje seriado de palabras). De esta forma, los pacientes que pueden tener una peor evolución oncológica según juicio clínico y/o los que padecen enfermedades crónicas adicionales no son necesariamente aquellos con más dificultades cognitivas. Por el contrario, los datos sugieren que los pacientes con un cáncer de colon en estadio III rinden peor que los de estadio II; la progresión de la enfermedad parece ser un factor de utilidad en la predicción de la afectación cognitiva. Este resultado va a favor de la hipótesis sobre la influencia adversa del aumento de las citoquinas producidas por el tumor en la cognición, apoyada por algunos estudios precedentes $^{(23-25)}$. Otro trabajo ${ }^{(26)}$, confirma que los pacientes, tanto los que recibieron quimioterapia como los que no, tienen las citoquinas más elevadas que las personas sanas y con una tendencia a peor rendimiento cognitivo; en 326 pacientes de cáncer de colon también se apreció una elevada cantidad de citoquinas respecto a las personas sanas antes de la quimioterapia, pero sin asociación significativa del estadio del cáncer y las citoquinas con la cognición ${ }^{(21)}$. Ante estos datos, no se descarta con certeza que las citoquinas influyan en el rendimiento cognitivo y nos preguntamos si otros factores asociados a la enfermedad pudieran influir en la cognición; por ello se recomienda cautela al respecto, siendo necesarios estudios que lo aclaren.

La calidad de vida global de la muestra se relaciona ligeramente con aprendizaje seriado de palabras de forma positiva. Cuando se analizan las áreas funcionales que abarca la calidad de vida global (física, rol, emocional, cognitiva y social) se encuentran claros resultados. Cuatro de las cinco áreas funcionales no se vinculan con el rendimiento cognitivo objetivo. La función social es la única que mantiene correlaciones con el rendimiento en los diferentes test de la habilidad psicomotora, de memoria verbal y en subpruebas de función ejecutiva (Palabra, Color del test Stroop y TMT-B). Es razonable que las personas con más dificultades necesiten, soliciten y/o reciban más sustento social, y es posible que se muestren más satisfechos con las conductas de apoyo recibidas, o incluso que los pacientes más afectados cognitivamente tengan otras preocupacio- 
nes que les hacen ser menos críticos con su calidad de vida social. Mientras, las personas con mejor rendimiento cognitivo, posiblemente jóvenes que soportan una mayor carga social (vida laboral activa, responsabilidades familiares), consecuentemente, podrían tener más problemas al llevarlas a cabo a raíz de la enfermedad, o incluso percibir y/o ser objeto de una mayor estigmatización social como paciente de cáncer.

Aunque pueda sorprender el dato que descarta una relación entre la percepción cognitiva del paciente y el funcionamiento presentado en las pruebas objetivas, dicha percepción parece estar más bien vinculada a la ansiedad y depresión que al propio rendimiento. Los pacientes que expresan quejas más intensas sobre sus dificultades en memoria y capacidad de concentración (área funcional cognitiva del QLQ-C30) puntúan en mayor medida en sintomatología ansiosa y depresiva. Algunos autores sugieren que las quejas cognitivas indican más bien malestar emocional; además, los pacientes que se quejaban más no eran necesariamente los que ostentaban deterioro cognitivo identificado ${ }^{(21,27)}$. Esto Ileva a plantear que la percepción subjetiva del paciente no se corresponde con el rendimiento objetivo. Las pruebas de autoinforme, tales como el QLQ-C30 pueden resultar escasamente útiles en la valoración de daño cognitivo.

El screening realizado sobre problemas físicos, revela que las dificultades respiratorias del paciente se relacionan con una menor función ejecutiva, habilidad psicomotora y memoria verbal (exceptuando la puntuación interferencia del test Stroop). Padecer dolor de mayor intensidad puede empeorar la memoria diferida, con o sin pistas. La fatiga referida se asocia con menor velocidad psicomotora (TMT-A) y peor recuerdo inmediato libre y diferido con pistas.

Por su parte, encontramos resultados en varias direcciones en relación a los problemas psicosociales, existiendo tanto correlaciones positivas como negativas. De un lado existiría una relación indicativa de déficit cognitivo asociado a dificultades psicosociales; de otro, es probable que las personas con mejor puntuación en algunas pruebas neuropsicológicas, sean más conscientes de algunos problemas psicosociales o de los desafíos que comportan, mientras que las personas con menores habilidades cognitivas, que suelen ser los de edad avanzada, podrían estar sometidas a menos estrés psicosocial (por ejemplo laboral) o considerar con mayor normalidad algunas fuentes de estrés. Dada la escasa congruencia entre datos, es prematuro establecer conclusiones firmes sobre estas relaciones, por ello, alentamos su estudio y replicación con el fin de esclarecer los vínculos entre estas variables.

\section{CONCLUSIÓN}

Según los datos extraídos, una primera aproximación al perfil de alto riesgo de daño cognitivo en pacientes de cáncer de colon tratados con cirugía, antes de la aplicación de quimioterapia sería: paciente de mayor edad, apreciándose daños en el rendimiento a partir de los 50 años, con cáncer de colon en estadio III, tiene menos de cinco años de estudios, y una alta percepción en su calidad de vida social, refiere problemas respiratorios (asma u otras dificultades respiratorias). Algunos pacientes presentan déficits cognitivos antes de recibir la quimioterapia. Estos deterioros previos pueden alcanzar el $33 \%$ de afectación en función ejecutiva, el $21 \%$ en habilidad psicomotora y el $11 \%$ en memoria verbal. Este dato nos indica que los estudios transversales pueden sobreestimar el deterioro asociado a quimioterapia pues ignoran el daño previo a la quimioterapia, y también que existen otro tipo de factores influyentes en 
la cognición que actúan antes de aplicar este tratamiento sistémico.

\section{REFERENCIAS BIBLIOGRÁFICAS}

1. Vardy J, Wong K, Yi Q, Park A, Maruff $\mathrm{P}$, Wagner $\mathrm{L}$, et al. Assessing cognitive function in cancer patients. Support Care Cancer, 2006; 14: 1111-18. Doi:10.1007/ s00520-006-0037-6

2. Rodin MB, Wallace JA, Lacy M, Kuball K, Pykkonen B, Freming G. Does adjuvant chemotherapy (CT) exacerbate cognitive impairment in elderly breast cancer (BC) patients? Results of a prospective, longitudinal study. J Clin Oncol 2006; 24, (20 Suppl): 10530.

3. Wefel JS, Saleeba AK, Buzdar AU, Meyers CA. Acute and late onset cognitive dysfunction associated with chemotherapy in women with breast cancer. Cancer, 2010; 116: 3348-56. Doi:10.1002/cncr.25098

4. Meyers CA, Byrne KS, Komaki R. Cognitive deficits in patients with small cell lung cancer before and after chemotherapy. Lung Cancer, 1995; 12: 231-5. Doi:10.1016/0169-5002(95)00446-8

5. Peña-Casanova J. Manual Test Barcelona Revisado Programa integrado de exposición neuropsicológica. Barcelona: Masson, 1990.

6. Peña-Casanova J. Normalidad, semiología y patología neuropsicológicas: programa integrado de exploración neuropsicológica "Test Barcelona". Barcelona: Masson, 1991

7. Peña-Casanova J. Programa integrado de exploración neuropsicológica "Test Barcelona" Normalidad, Semiología y Patología neuropsicológicas. Barcelona: Masson, 2001.

8. Peña-Casanova J, Gramunt NF, Gich JF. Test neuropsicológicos. Fundamentos para una neuropsicología clínica basada en evidencias. Barcelona: Masson, 2004.

9. Golden CJ. Stroop, Test de Colores y Palabras. Madrid: TEA; 2005.

10. Alsworth M. The Trail Making Test. En Long C), editor. Neuropsychology \& behavioral neuroscience, 1997. Extraído el 12 Abril de 2007 del sitio Web de Memphis University: http://neuro.psyc.memphis.edu/NeuroPsyc/ np-test1.htm\#trails

11. Poon M. Trail Making Test. [En línea] 2004. [Acceso el 7 de Julio, 2008]. Disponible en: http://www.angelfire.com/retro/michaelpoon168/trail_making_test.htm

12. Reitan RM. Validity of the Trail Making Test as an indicator of organic brain damage. Percept. Mot Skills 1958; 8: 271-6. Doi:10.2466/PMS.8.7.271-276

13. Fayers PM, Aaronson NK, Bjordal K, Groenvold M, Curran D, Bottomley A on behalf of the EORTC Quality of Life Group. EORTC QLQ-C30 Scoring Manual, 3rd ed. Brussels: EORTC, 2001.

14. Arrarás JI, Illarramendi JJ, Valerdi JJ. El cuestionario de Calidad de Vida de la EORTC, QLQ-C30. Estudio estadístico de validación con una muestra española. Rev Psicol Salud 1995; 7: 13-30.

15. Arrarás Jl, Dueñas T, Meiriño R, Prujá E, Villafranca E, Valerdi JJ. La calidad de vida en el paciente oncológico: estudios del Servicio de Oncología del Hospital de Navarra en el Grupo de Calidad de Vida de la EORTC. Anales, 1998; 21 (1). [En línea] [acceso el 23 de Abril, 2007]. Disponible en: http://www.cfnavarra.es/salud/anales/ textos/vol21/n1/revis2a.html

16. Arrarás Jl, Martínez M, Manterota A, Laínez N. La evaluación de la calidad de vida del paciente oncológico. El grupo de la calidad de vida de la EORTC. Psicooncología 2004; 1 (1): 87-98.

17. Zigmond AS, Snaith R P. The Hospital Anxiety and Depression Scale. Acta Psychiatr Scand 1983; 67: 361-70. Doi:10.1111/j.1600-0447.1983.tb09716.x

18. Caro I, Ibáñez E. La escala hospitalaria de ansiedad y depresión. Su utilidad práctica en Psicología de la salud. Bol Psicol 1992: 1(36): 43-69.

19. Mendoza TR, Wang XS, Cleeland CS, Morrisey M, Johnson BA, Wendt JK, et al. The rapid assessment of fatigue se- 
verity in cancer patients: Use of the Brief Fatigue Inventory. Cancer 1999; 85 (5): 1186-96. Doi:10.1002/(SICI)1097$0142(19990301) 85: 5<1186:$ :AID CNCR24>3.0.CO;2-N

20. Ordóñez A, Feijoo M, González Barón M. Evaluación de la astenia. En: GonzálezBarón M, Lacaste MA, Ordóñez A, editores. Valoración clínica en el paciente con cáncer. Buenos aires; Madrid: Médica panamericana, 2006; p. 79-87.

21. Vardy J, Dhillon H, Xu W, Dodd A, Park A, Rourke SB, et al. Cognitive function and fatigue in colorectal cancer (CRC) patients: Baseline assessments prior to chemotherapy. J Clin Oncol 2009; 27. No 15 S (20 Supplement), 9557.

22. Cimprich B, So H, Ronis DL, Trask C. Pre-treatment factors related to cognitive functioning in women newly diagnosed with breast cancer. Psychooncology, 2005; 14: 70-8. Doi:10.1002/pon.821

23. Argyriou A, Assimakopoulos K, Iconomou G, Giannakopoulou F, Kalofonos H. Either caller "chemobrain" or "chemofog", the long-term chemotherapy-induced cognitive decline in cancer survivors is real.
J Pain Symptom manage, 2010. Extraído el 17 de Octubre de 2010 del sitio Web: http://www.ncbi.nlm.nih.gov/pub$\mathrm{med} / 20832978$

24. Maier S, Watkins L. Immune-to-central nervous system communication and its role in modulating pain and cognition: implications form cancer and cancer treatment. Brain, Behav Imm 2003; 17: 125-31. Doi:10.1016/S0889-1591(02)00079-X

25. Meyers CA. How chemotherapy damages the central nervous system. J Biol 2008; 7:11. Doi:10.1186/jbiol73

26. Vardy J, Rourke S, Pond GR, Galica J, Park A, Dhillon $\mathrm{H}$, et al. Cognitive function and fatigue in cancer patients after chemotherapy: A longitudinal cohort study in patients with colorectal cancer (CRC). J Clin Oncol 2007; 25, No18S (20 Suppl): 9099.

27. van Dam FSAM, Schagen SB, Muller MJ, Boogerd W, Wall E, Droogleever MEF, et al. Impairment of cognitive function in women receiving adjuvant treatment for high-risk breast cancer: High-dose versus Standard-dose chemotherapy. J Nat Cancer Inst, 1998; 90: 210-18. Doi:10.1093/ jnci/90.3.210 